\title{
Erratum to: A retrospective study to identify risk factors for somnolence and dizziness in patients treated with pregabalin
}

Hiroshi Kato ${ }^{1,2^{*}}$, Masayuki Miyazaki ${ }^{1,2}$, Mio Takeuchi ${ }^{2}$, Hiroaki Tsukuura ${ }^{3}$, Mihoko Sugishita ${ }^{3}$, Yukihiro Noda $^{1,2}$ and Kiyofumi Yamada ${ }^{1}$

\section{Erratum}

Unfortunately, after publication of the original version of this article [1], it was noticed that some author corrections were not included in the final article.

- Within the "Methods" section, the Cockcroft-Gault equation should read as follows:

$$
\begin{aligned}
\operatorname{CLcr}(\mathrm{mL} / \mathrm{min})= & (140-\text { age }) \\
& \times \text { weight }(\mathrm{kg}) /\{72 \\
& \times \text { serum creatinine }(\mathrm{mg} / \mathrm{dL})\} \\
& \times 0.85(\text { if female })
\end{aligned}
$$

- In addition Table 1 contained some formatting errors. The correct version of Table 1 is included below.

\footnotetext{
* Correspondence: hk-hiroshi@med.nagoya-u.ac.jp

${ }^{1}$ Department of Neuropsychopharmacology and Hospital Pharmacy, Nagoya University Graduate School of Medicine, 65 Tsurumai-cho, Showa-ku, Nagoya, Aichi 466-8560, Japan

${ }^{2}$ Division of Clinical Sciences and Neuropsychopharmacology, Meijo

University Faculty of Pharmacy, Nagoya, Japan

Full list of author information is available at the end of the article
} 
Table 1 Patient characteristics $(n=204)$

\begin{tabular}{|c|c|}
\hline \multicolumn{2}{|l|}{ Age } \\
\hline Median, year & 61 \\
\hline Range, year & $18-92$ \\
\hline \multicolumn{2}{|l|}{ Gender } \\
\hline Male, $n(\%)$ & $123(60.3)$ \\
\hline Female, $n(\%)$ & $81(39.7)$ \\
\hline Median $\mathrm{CLCr}{ }^{\mathrm{a}}$, $\mathrm{mL} / \mathrm{min}$ & 88.7 \\
\hline \multicolumn{2}{|l|}{$N P^{b}$ origin } \\
\hline Cancer related NP, $n(\%)$ & $55(27.0)$ \\
\hline Chemotherapy related NP, $n(\%)$ & $24(11.7)$ \\
\hline Non-cancer related NP, n(\%) & $125(61.3)$ \\
\hline \multicolumn{2}{|l|}{ Regular strong opioid } \\
\hline yes, $n(\%)$ & $36(17.6)$ \\
\hline -fentanyl, $n$ & 17 \\
\hline -oxycodone, $n$ & 16 \\
\hline -morphine, $n$ & 3 \\
\hline Median dose ${ }^{c}$ (range), mg/day & $60(15-300)$ \\
\hline no, $n(\%)$ & $168(82.4)$ \\
\hline \multicolumn{2}{|l|}{ Initial dose of pregabalin } \\
\hline 150 mg/day, $n(\%)$ & $124(60.8)$ \\
\hline 75 mg/day, $n(\%)$ & 73 (35.8) \\
\hline 50 mg/day, $n(\%)$ & $3(1.5)$ \\
\hline 25 mg/day, n(\%) & $4(1.9)$ \\
\hline
\end{tabular}

${ }^{\mathrm{a}} \mathrm{CLcr}$ : creatinine clearance using the Cockcroft-Gault equation

${ }^{\mathrm{b}} \mathrm{NP}$ : neuropathic pain

converted to oral morphine

\section{Author details}

'Department of Neuropsychopharmacology and Hospital Pharmacy, Nagoya University Graduate School of Medicine, 65 Tsurumai-cho, Showa-ku, Nagoya, Aichi 466-8560, Japan. ${ }^{2}$ Division of Clinical Sciences and

Neuropsychopharmacology, Meijo University Faculty of Pharmacy, Nagoya, Japan. ${ }^{3}$ Department of Clinical Oncology and Chemotherapy, Nagoya

University Hospital, Nagoya, Japan.

Received: 11 February 2016 Accepted: 11 February 2016

Published online: 17 February 2016

\section{References}

1. Kato H, Miyazaki M, Takeuchi M, Tsukuura H, Sugishita M, Noda Y, et al. A retrospective study to identify risk factors for somnolence and dizziness in patients treated with pregabalin. J Pharm Health Care Sci. 2015;1:22. doi:10.1186/s40780-015-0022-7.

Submit your next manuscript to BioMed Central and we will help you at every step:

- We accept pre-submission inquiries

- Our selector tool helps you to find the most relevant journal

- We provide round the clock customer support

- Convenient online submission

- Thorough peer review

- Inclusion in PubMed and all major indexing services

- Maximum visibility for your research

Submit your manuscript at www.biomedcentral.com/submit 